\title{
Desarrollo del pensamiento espacial en estudiantes del preuniversitario
}

\section{Development of spatial thinking in high school students}

1 Jorge Luis Puyol-Cortez

iD https://orcid.org/0000-0002-0734-694X

Universidad Técnica Luis Vargas Torres de Esmeraldas, Esmeraldas, Ecuador,

Maestría en Matemática mención Modelación y Docencia

jorge.puyol@utelvt.edu.ec

2 Julio César Rivadeneira-Moreira

https://orcid.org/0000-0003-4889-4392

Universidad Técnica Luis Vargas Torres de Esmeraldas, Esmeraldas, Ecuador, Sede Santo Domingo

julio.rivadeneira.moreira@utelvt.edu.ec

3 Holger Arturo Intriago-Mairongo https://orcid.org/0000-0003-3694-1219

Universidad Técnica Luis Vargas Torres de Esmeraldas, Esmeraldas,

Ecuador, Sede Santo Domingo

holger.intriago@utelvt.edu.ec

$4 \quad$ Nilo Alberto Benavides-Solís

https://orcid.org/0000-0002-0885-708X

Universidad Técnica Luis Vargas Torres de Esmeraldas, Esmeraldas, Ecuador,

Maestría en Matemática mención Modelación y Docencia

nilo.benavides@utelvt.edu.ec

Artículo de Investigación Científica y Tecnológica
Enviado: 07/01/2022
Revisado: $20 / 01 / 2022$
Aceptado: 17/02/2022
Publicado:07/03/2022
DOI: https://doi.org/10.33262/ap.v4i1.190

DOI: https://doi.org/10.33262/ap.v4i1.190

Solís, N. A. (2022). Desarrollo del pensamiento espacial en estudiantes del

Cítese: $\quad$ preuniversitario. AlfaPublicaciones, 4(1), 206-221. https://doi.org/10.33262/ap.v4i1.190

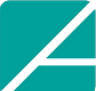

Ciencia

Digital

ALFA PUBLICACIONES, es una Revista Multidisciplinar, Trimestral, que se publicará en soporte electrónico tiene como misión contribuir a la formación de profesionales competentes con visión humanística y crítica que sean capaces de exponer sus resultados investigativos y científicos en la misma medida que se promueva mediante su intervención cambios positivos en la sociedad. https://alfapublicaciones.com

La revista es editada por la Editorial Ciencia Digital (Editorial de prestigio registrada en la Cámara Ecuatoriana de Libro con No de Afiliación 663) www.celibro.org.ec

Esta revista está protegida bajo una licencia Creative Commons Attribution Non Commercial No Derivatives

4.0 International. Copia de la licencia: http://creativecommons.org/licenses/by-nc-nd/4.0/ 
Palabras claves: matemáticas, pensamiento espacial, comprensión

Keywords:

mathematics, spatial thinking, comprehension.

\section{Resumen}

Durante el transcurso de los tiempos la comprensión de la matemática fue, es y será el principal temor de la gran mayoría de estudiantes de cualquier edad. El objetivo de la investigación es el de analizar el pensamiento espacial de los estudiantes bajo una aplicación de estrategias del algebra geométrica a dos colores. Este trabajo se basa en una revisión bibliográfica bajo un enfoque descriptivo cualitativo donde se realiza una revisión minuciosa de artículos científicos relacionados con la temática en mención. El conocimiento de la teoría de las inteligencias múltiples de Gardner para centrar esta investigación en una de sus más relevantes ideas, la de la Inteligencia Espacial, la misma que se refiere a la capacidad que tienen los individuos de comprender su realidad circundante por medio de figuras, objetos, espacios, y con ello visualizar mentalmente la construcción de formas que representan los conceptos, comprenderlos y encontrar varias alternativas de solución. Aplicando esta idea a la comprensión de la matemática, se propone que el educando mire a los ejercicios y problemas matemáticos desde una óptica más realista y de fácil asimilación. Este estudio permitirá hacer la comparación del enfoque y su contribución para alcanzar el desarrollo del pensamiento espacial, observando los resultados en la implementación de estrategias como el álgebra geométrica a dos colores, la realidad aumentada y los ambientes virtuales remotos para el aprendizaje, que demuestren que se aumenta significativamente el rendimiento académico.

Abstract
During time the comprehension of mathematics was, is and will be
the main fear of the great majority of students of any age. The
objective of the research is to analyze the spatial thinking of
students under an application of geometric algebra strategies to two
colors. This work is based on a bibliographic review under a
qualitative descriptive approach where a thorough review of
scientific articles related to the topic in question is carried out. The
knowledge of Gardner's theory of multiple intelligences to focus
this research on one of his most relevant ideas, that of Spatial
Intelligence, which refers to the ability of individuals to understand
their surrounding reality through figures, objects, spaces, and thus
mentally visualize the construction of forms that represent the
concepts, understand them, and find several alternative solutions.

\section{Abstract}

During time the comprehension of mathematics was, is and will be the main fear of the great majority of students of any age. The objective of the research is to analyze the spatial thinking of students under an application of geometric algebra strategies to two colors. This work is based on a bibliographic review under a qualitative descriptive approach where a thorough review of scientific articles related to the topic in question is carried out. The this research on one of his most relevant ideas, that of Spatial Intelligence, which refers to the ability of individuals to understand their surrounding reality through figures, objects, spaces, and thus concepts, understand them, and find several alternative solutions. 


\begin{abstract}
Applying this idea to the understanding of mathematics, it is proposed that the learner looks at mathematical exercises and problems from a more realistic and easily assimilated point of view. This study will allow the comparison of the approach and its contribution to achieve the development of spatial thinking, observing the results in the implementation of strategies such as two-color geometric algebra, augmented reality, and remote virtual environments for learning, showing that academic performance is significantly increased.
\end{abstract}

\title{
Introducción
}

La comprensión de la matemática ha sido un punto crítico en el proceso de enseñanza aprendizaje, la mayoría de los estudiantes generalmente presentan una aversión o resistencia considerándola como una asignatura difícil y poco comprensible; así mismo existen alumnos que desarrollan ciertas capacidades que les permite asimilar los conocimientos de una forma más simple que los demás, estos últimos desarrollan el pensamiento espacial.

La teoría de las inteligencias múltiples de Gardner (1993), ha tomado gran importancia para la comprensión del proceso de enseñanza - aprendizaje, esto ha permitido a los docentes desarrollar diferentes estrategias y técnicas para poder impartir los conocimientos de forma óptima. Una de ellas es la inteligencia espacial, Gardner la describe como esencial para el desarrollo del pensamiento científico, puesto que permite manipular mediante la representación de información en forma física los problemas matemáticos (Medina et al., 2019), de esta manera se puede desarrollar el aprendizaje en la resolución de problemas de forma más realista que simplemente leyéndolo y resolviéndolo sobre una hoja.

La inteligencia espacial es desarrollada por ciertas personas durante su formación académica, y está relacionada con el desarrollo de destrezas como la abstracción espacial, la visualización y el pensamiento espaciales, estas habilidades permiten a los estudiantes comprender de forma eficiente y efectiva la matemática.

Es necesario que la enseñanza de la matemática evolucione; la sociedad ha evolucionado a través de los años y esto ha conllevado al desarrollo de nuevas tecnologías, a tal punto que dependemos en gran medida al desarrollo tecnológico para comunicarnos, aprender y facilitarnos ciertas actividades. Es por esta razón que no se puede continuar enseñando las matemáticas de forma tradicional, puesto que limita el desarrollo de las habilidades espaciales (Olivo \& Corrales, 2020), presentando a los problemas de forma teórica para 
que sean resueltos por el alumno sobre un papel lo que genera un desinterés, la percepción de dificultad e impide la comprensión de la asignatura por parte del estudiante.

Durante el año 2020 la humanidad se enfrentó a una pandemia por COVID-19, lo que obligó a algunos países, entre ellos Ecuador, a tomar medidas extremas como lo fue entrar en cuarentena en donde se suspendieron todas las actividades presenciales migrando al desarrollo de trabajo y estudio en la modalidad virtual (Zurita et al., 2020), esto representó tanto para los docentes como para los estudiantes un nuevo reto; enfrentándonos a una realidad que era latente, que consistía en que el proceso enseñanza - aprendizaje ya no podía continuar desarrollándose de la forma tradicional y que ahora los seres humanos necesitan de nuevas formas, métodos, técnicas y herramientas para aprender.

El presente artículo busca analizar las bases conceptuales y las diferentes herramientas tradicionales y tecnológicas existentes que le permitirán a los alumnos desarrollar habilidades espaciales, en la resolución y aplicación de ejercicios matemáticos, de forma que no solamente sean capaces de visualizar los problemas de forma tridimensional, sino que sean capaces de resolverlos utilizando las matemáticas; para ello se utilizó una metodología descriptiva - exploratoria que consiste en la estructuración de un marco conceptual en donde se revisará la literatura, estructurándola desde las concepciones hasta los instrumentos que se pueden utilizar para medir los resultados en la aplicación de las técnicas descritas (Herrera-Enríquez et al., 2021b), finalmente se compararán los enfoques cualitativos y cuantitativos de las diferentes técnicas sujetas a análisis y los resultados obtenidos en los estudios analizados, emitiendo un criterio técnico - científico sobre el grado de efectividad de las mismas en el desarrollo del pensamiento espacial y rendimiento académico.

Se analizarán herramientas como son el álgebra geométrica a dos colores, la realidad aumentada y los ambientes virtuales remotos para el aprendizaje. Se compararán los diferentes estudios que sustentan estas herramientas, se analizarán sus aplicaciones y se compararán sus resultados, para identificar cuál brinda un mejor resultado en el proceso de enseñanza - aprendizaje en la asignatura de matemáticas y que ofrezca un desarrollo del pensamiento espacial en el mayor número de estudiantes del preuniversitario.

\section{Metodología}

Con la finalidad de exponer objetivamente la metodología aplicada en el desarrollo del presente artículo se describirá el proceso realizado en donde se partió, desde el desarrollo de bases teórico - conceptuales comparativas, hasta la comparación de los resultados reflejados en estudios realizados en donde se aplicaron las técnicas para el desarrollo del pensamiento espacial (Herrera-Enríquez et al., 2021a); de forma que se pueda comparar los resultados obtenidos con la aplicación de las diferentes herramientas para determinar 
la que presente un mayor impacto en la comprensión de la asignatura, así como el incremento del desempeño académico.

Se realizó una revisión descriptiva con la finalidad de confrontar los conceptos, aplicaciones y estudios realizados de las diferentes técnicas (Merino, 2011), para ello se analizó exhaustivamente la bibliografía que demuestra la relevancia de la aplicación de las herramientas para lograr un mejor rendimiento académico. Se utilizó la base de datos de Google Académico usando descriptores como "Álgebra geométrica", "Realidad aumentada", “Ambientes virtuales remotos", "herramientas 3D”, "impresiones en 3D", "razonamiento espacial", "matemáticas", "cálculo" y "teoría de las inteligencias múltiples" siendo las palabras claves que fundamentaban la búsqueda; se hallaron alrededor de cincuenta artículos los cuales fueron clasificados de acuerdo con su relevancia y resultados obtenidos en la aplicación de las técnicas sujetas a análisis.

Para elegir los artículos que forman parte del presente estudio se analizaron parámetros como la calidad de la metodología aplicada, la relevancia de los resultados, la identificación de los hallazgos comunes entre los artículos que implican una misma técnica, para poder realizar la comparación y contrastación de los resultados mostrados en los estudios (López et al., 2021).

En el desarrollo se presenta la información más importante encontrada en los artículos como conceptualizaciones, metodologías aplicadas y resultados arrojados por los diferentes estudios, abordándolos desde los temas generales hasta las técnicas sujetas a análisis; finalmente en la discusión se encuentra una argumentación analítica de los resultados obtenidos y su influencia en el desarrollo del pensamiento espacial, así como su contribución relevante en la mejora del rendimiento académico en asignaturas derivas de las matemáticas (Sánchez et al., 2021).

\section{Resultados y Discusión}

\section{Inteligencias múltiples}

La teoría de las inteligencias múltiples de Gardner (1993), ha tomado gran importancia para la comprensión del proceso de enseñanza - aprendizaje, esto ha permitido a los docentes desarrollar diferentes estrategias y técnicas para poder impartir los conocimientos de forma óptima. Una de ellas es la inteligencia espacial, Gardner la describe como esencial para el desarrollo del pensamiento científico, puesto que permite manipular mediante la representación de información en forma física los problemas matemáticos (Medina et al., 2019), de esta manera se puede desarrollar el aprendizaje en la resolución de problemas de forma más realista que simplemente leyéndolo y resolviéndolo sobre una hoja. 


\section{Inteligencia espacial}

La inteligencia espacial es desarrollada por ciertas personas durante su formación académica, ésta está relacionada con el desarrollo de destrezas como la abstracción espacial, la visualización y el pensamiento espaciales, estas habilidades permiten a los estudiantes comprender de forma eficiente y efectiva la matemática.

La idea de la existencia de diferentes habilidades espaciales siempre ha estado en discusión e incluso ha sido clasificada bajo diferentes enfoques por algunos autores, entre ellos Gardner, Linn y Petersen y otros más. Gardner (1993), menciona que:

El propio Thurstone dividía la habilidad espacial en tres componentes: la habilidad para reconocer la identidad de un objeto cuando se ve desde ángulos distintos; la habilidad de imaginar el movimiento o desplazamiento interno entre las partes de una configuración, y la habilidad para pensar en las relaciones. (p. 142)

Como se evidencia se puede diferenciar tres componentes bien definidos, aquellas que llamaremos habilidades espaciales. Otra idea que también se considera importante es la expuesta por Medina et al. (2019), quienes citan a Gardner (1993), "la inteligencia espacial comprende una cantidad de capacidades relacionadas de manera informal: la habilidad para reconocer instancias del mismo elemento; la capacidad de evocar la imaginería mental y luego transformarla" (p. 41), Linn y Petersen (1985) fueron quienes definieron los términos y las estudiaron con mayor profundidad diferenciando la facilidad en la compresión del pensamiento espacial y el desarrollo de habilidades espaciales de acuerdo al género de los alumnos.

\section{Técnicas para el desarrollo del pensamiento espacial}

Howard Gardner (1993), en su teoría de las inteligencias múltiples señala como una de estas inteligencias la espacial, y plantea que es esencial para el pensamiento científico porque la misma es utilizada expresar y manipular información para aprender y resolver problemas en las diferentes áreas de su aplicación. Dentro de las utilizaciones de la información espacial resalta el resolver problemas de ubicación, orientación y distribución de espacios, actividades que son peculiares en esas personas que tienen desarrollada su inteligencia espacial (2000). Las estimaciones de la mayoría de las industrias científicas y técnicas, como el dibujo técnico, la arquitectura, la ingeniería, la aviación y muchas industrias científicas, como la química, la física, las matemáticas, se requieren personas con un amplio desarrollo de la inteligencia espacial. Se analizarán herramientas como son el álgebra geométrica a dos colores, la realidad aumentada y los ambientes virtuales remotos para el aprendizaje 
Pulgarin et al. (2019), describen el término de álgebra geométrica como operaciones geométricas definidas sobre segmentos de rectas o áreas planas las cuales tienen las mismas propiedades de suma y multiplicación de números reales, siendo la principal relación la igualdad sujeta a tres axiomas, utiliza un sistema simbólico de figuras geométricas como cuadrados y rectángulos como soporte, actuando como instrumento que permite facilitar el proceso de enseñanza - aprendizaje.

Para Palacios et al. (2018), el álgebra geométrica es un método de aplicación entre un segmento de recta y un área en donde se construye una parte de la longitud igual al área dada. De dicha afirmación se pueden obtener tres resultados el primero es que, si el segmento sea igual al primero, se indica que el área se aplica sobre el segmento; el segundo cuando el segmento es menor al primero se indica que es corto y finalmente si el segmento es mayor al primero se indica que éste se excede.

En un estudio realizado a estudiantes de bachillerato por Guerra (2018), el cual aplica la técnica del álgebra geométrica a dos colores elaborando figuras planas como cuadrados y rectángulos con diferentes tamaños y áreas; con cartulinas de doble color en donde se designó a un color para números positivos y otro color para números negativos, repitiéndose estos patrones en las demás áreas.

Una complejidad latente en el proceso de enseñanza - aprendizaje de las matemáticas consiste en el traspaso de la aritmética hacia el álgebra, puesto que implica una nueva óptica de las matemáticas para el alumno desarrollando su pensamiento lógico (Arreaza $\&$ Carvajal, 2013). Esto genera que los alumnos entren, de cierto modo, en una especie de colapso ya que debían de desarrollar el pensamiento lógico migrándolo de los números naturales al algebraico en donde deben de aprender a interpretar expresiones algebraicas. Para Arreaza \& Carvajal (2013), una respuesta a este problema es utilizar la geometría para ayudar a que la migración del pensamiento sea menos compleja, utilizando para ello los bloques de Dienes, éstos consisten en cuadrados grandes y pequeños y rectángulos de ciertas dimensiones, es decir es otro modo de denominar al álgebra geométrica.

La investigación realizada por Rivera (2020), a 23 estudiantes del $8^{\circ}$ grado, quien aplicó un enfoque de investigación - acción, desarrollando habilidades de motricidad fina al momento en que los alumnos elaboraron su material didáctico del álgebra geométrica a dos colores; existió la participación colaborativa por los sujetos de estudio, se establecieron relaciones entre el álgebra y la geometría, se observó el desarrollo del pensamiento espacial en la ejecución de la resolución de ejercicios prácticos por parte de los alumnos, quienes captaron satisfactoriamente el concepto de expresión algebraica, se logró que los alumnos relacionen el lenguaje común con el aritmético, algebraico y geométrico para la resolución satisfactoria de problemas matemáticos. 
De acuerdo con la investigación realizada por Palacios et al. (2018), a los estudiantes del $9^{\circ}$ grado de una unidad educativa, aplicando métodos cualitativos y cuantitativos, y utilizando técnicas de recolección de datos como la observación, lista de control, prueba estandarizada, diario pedagógico y plan de trabajo, cuya información respaldan los resultados obtenidos, los estudiantes respondieron de forma asertiva en la aplicación del álgebra geométrica a dos colores, existió una disminución sustancial en el tiempo de respuesta, obteniendo mejores resultados, finalmente se logró que los estudiantes relacionen los conceptos con los contextos aplicables en problemas reales.

\section{Realidad aumentada en cálculo (ARC)}

Mamani (2014,) la describe como una definición de visión directa o indirecta de un entorno físico mundial real, hay factores combinados con elementos virtuales para crear factores reales en tiempo real. Esta nueva tecnología completa la conciencia y la interacción con el mundo real y permite a los usuarios estar en un entorno realmente mejorado con información adicional creada por las computadoras.

Una de las habilidades del pensamiento espacial es la de visualización espacial, el desarrollo de las tecnologías de la información como la realidad aumentada, ha permitido tomar un objeto inanimado y que éste se vuelva tridimensional dando como resultado una visualización dinámica lo que genera el desarrollo de un pensamiento especializado para la resolución de ejercicios matemáticos de forma más sencilla (Medina et al. 2019).

Para Martínez et al. (2021), la realidad aumentada constituye un desarrollo tecnológico en donde se superpone a una imagen real una imagen en 3D o cualquier otra información que sea generada por un dispositivo tecnológico, para poder aplicarla exitosamente es necesario contar con ciertos aspectos técnicos, funcionales, avances y adaptación por parte tanto de docentes como de alumnos. En el campo de las matemáticas la aplicación de esta técnica les permite a los estudiantes manipular la realidad de los conceptos establecidos de forma teórica y aplicarlos exitosamente, aumentado sustancialmente el interés.

Bujak et al. (2013), abordan la ARC desde tres dimensiones: la física mediante la interacción de los objetos representados físicamente para la comprensión de conceptos, la cognitiva para la comprensión de conceptos abstractos mediante la alienación espacio tiempo y contextual en donde se analiza la importancia de la ARC en el aprendizaje colaborativo que general experiencias significativas.

En un estudio realizado por Gibelli et al. (2017), establecen la importancia de una combinación adecuada entre software y hardware para poder aplicar adecuadamente una ARC; puesto que ésta permite capturar e identificar la escena real, fusión de la realidad y la virtualidad y la visualización de la imagen aumentada, además comparan dos softwares 
de realidad aumentada como son Blender y SketchUp dando unos mejores resultados la aplicación del software Blender.

Reyes (2020), manifiesta en su estudio que para una aplicación adecuada de la ARC en el proceso de enseñanza - aprendizaje es necesario que el docente tenga competencias digitales para poder aplicar efectivamente esta herramienta y poder alcanzar con éxitos los objetivos de aprendizaje planificados en el esquema curricular de la asignatura, además concluye que en base a las opiniones recolectadas por los estudiantes se puede afirmar que existe un alto grado de captación de los conocimientos cuando se utiliza la ARC

Para Salinas et al. (2013), la ARC es una alternativa atractiva para facilitar la comprensión de las matemáticas desde una perspectiva más real para los alumnos, mejorando significativamente el interés de los estudiantes en la asignatura desarrollando su habilidad de visualización espacial. La ARC funciona como un puente de comprensión que conecta la aplicación práctica - didáctica con los fundamentos conceptuales de las matemáticas activando los procesos cognitivos de los alumnos con la guía del docente.

La realidad aumentada permite integrar señales realistas con información generada por dispositivos tecnológicos, coexistiendo el mundo virtual con la realidad, permite construir el procesamiento de imágenes para extraer propiedades geométricas. Un sistema de realidad aumentada registra señales del mundo real que son procesadas por el sistema dando realce a las orillas de los objetos para segmentar la imagen reconociendo patrones y marcas esto permite la aplicación en varias ciencias entre ellas la matemática (Heras \& Villarreal, 2004).

Según un estudio realizado por Martínez et al. (2021), a 70 estudiantes de $9^{\circ}$ grado de educación básica de instituciones educativas de Colombia, donde se aplicó un estudio cuantitativo - explicativo cuasi experimental con dos grupos, uno de control y el otro experimental, se encontró que el grupo experimental logró superar el promedio del grupo de control debido a un aumento en el grado de motivación, además se registró un avance significativo entre el conocimiento superficial y el profundo.

La apreciación de 192 estudiantes de bachillerato que cursaban el primer semestre de la asignatura de Fundamentos de Matemáticas, cuyas opiniones fueron recolectadas y presentadas en el estudio realizado por George (2020), en donde se revelaron mejoras significativamente en la aprobación escolar en comparación con cursos anteriores, existió una acogida favorable de la realidad aumentada por parte de los alumnos mostrando un interés extraordinario en la asignatura, favoreciendo satisfactoriamente el desempeño de los estudiantes, es necesario trabajar en la adquisición de competencias digitales por parte del docente para crear vínculos entre las estrategias de enseñanza y la utilización de herramientas tecnológicas (Casanova-Villalba et al., 2022). 
En el estudio realizado por Pedraza \& Valbuena (2014), de una muestra de estudiantes que cursan las asignaturas de cálculo diferencial, integral y vectorial, bajo un enfoque cuantitativo que arrojo los siguientes resultados: los estudiantes aprendieron de manera autónoma, se ha eliminado las limitaciones de tiempo y espacio de las metodologías de enseñanza tradicionales, se logró una mejor comprensión de los conceptos teórico prácticos estudiados en las asignaturas y permitió la mejora del rendimiento académico.

De acuerdo con el estudio realizado por Berumen et al. (2021), en donde se realizó un estudio cuasi experimental en alumnos de la carrera de Contador Público del Tecnológico Nacional de México en la asignatura de Cálculo diferencial e integral, los resultados arrojaron que no existe diferencia en el proceso de enseñanza - aprendizaje entre el grupo que utilizó la herramienta de la realidad aumentada y el grupo que recibió las clases de forma tradicional, sin embargo si se encontró que los estudiantes que utilizaron la ARC experimentaron un mayor interés en el transcurso de la asignatura.

En el estudio realizado por Buitrago (2015), en donde participaron 83 estudiantes de la carrera de Ingeniería Industrial, la investigación se realizó cuantitativamente con un alcance descriptivo correlacional, arrojando los siguientes resultados: el uso de la ARC ha permitido obtener resultados favorables y satisfactorios para el logro de los objetivos de aprendizaje de la asignatura de cálculo vectorial, sin embargo la realidad aumentada por sí sola no conduce a desarrollar un conocimiento lógico, pero si es una herramienta importante que se puede utilizar para facilitar el desarrollo del pensamiento espacial en la solución de problemas matemáticos de cálculo considerando las características cognoscitivas de cada alumno.

\section{Los ambientes virtuales remotos para el aprendizaje de matemáticas (AVRAM)}

Medina \& Hernández (2013), indica que un AVRAM permite visualizar y manipular superficies en un espacio tridimensional virtual. Se presentan las características de AVRAM y las primeras pruebas con diferentes grupos de estudiantes y profesores en Matemáticas II y Matemáticas III. Se ha evidenciado que AVRAM es un instrumento importante para el desarrollo de las habilidades espaciales de los adoctrinados y la comprensión de conceptos relacionados con el espacio tridimensional.

Los ambientes virtuales permiten interactuar con objetos inanimados creados con la finalidad de explorar un pensamiento visual espacial construyendo una nueva realidad utilizando para ello las tecnologías que se encuentran al alcance de los usuarios para el desarrollo interactivo del proceso de enseñanza - aprendizaje ampliando sus capacidades mentales, considerando que lo virtual no sobrepase lo real (Esteban et al., 2006).

La influencia del docente en el proceso cognitivo de los alumnos a implicado el desarrollo de distintas herramientas de enseñanza, actualmente las Tecnologías de la Información y 
Comunicación, así como el internet ha cambiado radicalmente la concepción del proceso del desarrollo del pensamiento lógico tradicional, esta nueva interacción ha sido objeto de estudio para la enseñanza - aprendizaje de la matemática (Olivo \& Corrales, 2020).

En un estudio realizado por Zuluaga et al. (2015), menciona que ciertas ventajas y desventajas en el uso de ambientes virtuales de aprendizaje, entre las ventajas menciona "disminución de las limitaciones debidas a los déficits cognitivos, sensoriales y motores de los sujetos; además disminuyen el tiempo de adquisición de habilidades y destrezas" (p. 6) y como desventajas menciona "falta de recursos tales como acceso a internet y dispositivos como computadoras, teléfonos inteligentes o tabletas...problemas éticos" (p. 7).

Zurita et al. (2020), considera que los ambientes virtuales del aprendizaje han evolucionado significativamente el desarrollo del proceso enseñanza - aprendizaje, lo definen como "sistemas basados en internet" (p. 34) los cuales mantienen varias herramientas pedagógicas impulsando la educación de manera virtual, otro aspecto que ha impulsado los AVRAM ha sido la nueva normalidad dada por la pandemia que ha sufrido el mundo en los últimos años.

En cambio, para Martínez et al. (2005), un ambiente virtual de aprendizaje es una interfaz que permite interactuar e implementar el proceso de enseñanza - aprendizaje en un ambiente utilizando dispositivos tecnológicos considerando las implicaciones en el desarrollo educativo para ello se debe de conocer los recursos tecnológicos que disponen tanto los docentes como los alumnos y relacionarlos adecuadamente con los contenidos de la asignatura y el objetivo de estudio que se desea lograr.

De acuerdo con los resultados arrojados en el estudio realizado por Esteban et al. (2006), en donde se determina que los AVRAM permiten integrar exitosamente los conocimientos teóricos de los alumnos, pero depende significativamente del docente y el uso que les dé a estas herramientas en la impartición de conocimientos, para ello se debe diseñar metodologías apropiadas que motiven a los estudiantes a aprender, comprender y aplicar los conocimientos matemáticos.

Medina \& Hernández (2013), en su estudio AVRAM: Ambientes Virtuales Remotos para el Aprendizaje de las Matemáticas (2013), realizado con estudiantes y maestros de las asignaturas Matemáticas II y Matemáticas III; ha arrojado los siguientes resultados: se ha logrado el desarrollo de las habilidades espaciales y la concepción de los conceptos teóricos - conceptuales de las matemáticas en conjunción al espacio tridimensional, simplificando las explicaciones utilizando la metodología de enseñanza - aprendizaje tradicional. 
En un estudio realizado por, cuya muestra fueron 12 estudiantes de $9^{\circ}$ grado jornada tarde, en donde se aplicó un enfoque cuantitativo - experimental combinando metodologías pre - post y experimental - control, demostró que se desarrollan mejores capacidades en el proceso enseñanza - aprendizaje de los conceptos matemáticos, permitió a los estudiantes mejorar sustancialmente el pensamiento lógico y espacial, constituye una herramienta metodológica ideal para el docente y el alumno puesto que permite el acceso a la información en cualquier momento fortaleciendo la construcción de competencias y habilidades cognoscitivas (Herrera-Sánchez et al., 2022).

\section{Conclusiones}

- Existen pocos estudios de las aplicaciones prácticas de las técnicas elegidas en estudiantes universitarios lo que hace compleja realizar la tarea de comparar las herramientas, puesto que los pocos estudios encontrados son realizados con estudiantes con rangos de edades diferentes y con grados académicos que van desde la escolaridad hasta la universidad.

- En todos los estudios analizados, los autores enfatizan en que, para tener éxito en la aplicación de cualquier técnica, es indispensable el compromiso que realice el docente en mejorar sus habilidades digitales y migrar de una enseñanza tradicional a una nueva metodología de enseñanza - aprendizaje que incluya el uso de tecnología, a pesar de que existan estudios que avalen los excelentes resultados en desempeño académico.

- De acuerdo con los estudios analizados la herramienta que brinda los mejores resultados en el desarrollo del pensamiento espacial e incremento sustantivo en el rendimiento académico, son los ambientes virtuales del aprendizaje; puesto que engloba el uso de aplicaciones que permiten la visualización en tercera dimensión de objetos inanimados, haciendo uso de laboratorios virtuales, aplicaciones e impresiones en 3D.

\section{Referencias Bibliográficas}

Arreaza, T., \& Carvajal, E. (2013). Aritmética y álgebra a través de los bloques de dienes. Instituto Pedagógico de Caracas, 10.

Berumen, E., Acevedo, S., \& Raveles, S. (2021). Realidad aumentada como técnica didáctica no ensino de disciplinas de cálculo no ensino superior. Estudio de caso. Revista Iberoamericana para la Investigación y el Desarrollo Educativo, 26.

Buitrago, R. (2015). Incidencia de la realidad aumentada sobre el estilo cognitivo: caso para el estudio de las matemáticas. Universidad de la Sabana, 16. 
Bujak, K. R., Radu, I., Catrambone, R., Macintyre, B., Zheng, R., \& Golubski, G. (2013). A psychological perspective on augmented reality in the mathematics classroom. Computers \& Education, 68.

Casanova-Villalba, C. I., Herrera-Sánchez, M. J., Herrera-Enríquez, G., Almeida-Blacio, J. H., \& Preciado-Ortiz, F. L. (2022). Estrategia y Ventaja Competitiva-Un enfoque práctico.

Esteban, P., Trefftz, H., \& Restrepo, J. (2006). Estrategias de visualización en el cálculo de varias variables. Revista Educación y Pedagogía, 13.

Gardner, H. (1993). Marcos de la mente: la teoría de las inteligencias. Nueva York: BasicBooks.

George, C. (2020). Percepción de los estudiantes de bachillerato sobre el uso de Metaverse en experiencias de aprendizaje de realidad aumentada en matemáticas. Píxel-BIT Revista de Medios y Educación, 17.

Gibelli, T., Graziani, A., \& Sanz, C. (2017). Revisión de herramientas para la creación de modelos 3D orientados a la enseñanza de la matemática con realidad aumentada. Congreso Argentino de Ciencias de la Computación, 10.

Guerra, M. (2018). Factorización con geometría a dos colores. Universidad Nacional de Educación, 47.

Heras, L., \& Villarreal, J. (2004). La realidad aumentada: una tecnología en espera de usuarios. Revista Digital Universitaria, 9.

Herrera-Enríquez, G., Castillo Páez, S., Zambrano Vera, D., Herrera Sánchez, M. J., \& Casanova Villalba, C. I. (2021a). Incidencia de las metodologías de enseñanza en las carreras de ciencias administrativas ofertadas por las universidades públicas del DMQ. Visionario Digital, 20.

Herrera-Enriquez, G., Herrera-Sánchez, M., Casanova-Villalba, C., Puyol-Cortez, J., \& Mendoza-Armijos, H. (2021b). Manual para Elaboración del Plan de Titulación como Conclusión de Carrera. Grupo Compás.

Herrera-Sánchez, M. J., Casanova-Villalba, C. I., Herrera-Enríquez, G., Preciado-Ortiz, F. L., \& Bravo-Bravo, I. F. (2022). Estrategia y Ventaja Competitiva-Compilación y Análisis.

López, S. E. R., Villalba, C. I. C., Sánchez, M. J. H., \& Zambrano, C. M. N. (2021). Modelo interno para el aseguramiento de la calidad educativa con enfoque MPVA 
en el marco de las unidades productivas de las Instituciones de Educación Superior (IES) en Ecuador. ConcienciaDigital, 4(1), 34-47.

Mamani, J. (2014). Realidad aumentada para el desarrollo del pensamiento espacial. La Paz: Universidad Mayor de San Andrés.

Martínez, E., Arrieta, J. L., \& Canul, A. (2005). Laboratorio Virtual de Matemáticas. Instituto Tecnológico de Acapulco, 6.

Martínez, O. M., Mejía, E., Ramírez, W. R., \& Rodríguez, T. D. (2021). Incidencia de la realidad aumentada en los procesos de aprendizaje de las funciones matemáticas. Información Tecnológica, 12.

Medina, L., \& Hernández, B. (2013). AVRAM: Ambientes Virtuales Remotos para el Aprendizaje de las Matemáticas. Tecnológico de Monterrey, 5.

Medina, L., Castro, J., \& Juárez, S. (2019). Desarrollar habilidades matemáticas espaciales a través de herramientas 3D: realidad aumentada, entornos virtuales e impresión 3D. Revista internacional de diseño y fabricación interactivos, 15.

Merino, A. (2011). Como escribir documentos científicos (Parte 3). Artículo de revisión. Salud en Tabasco, 36 - 40.

Olivo, J., \& Corrales, J. (2020). De los entornos virtuales de aprendizaje: hacia una nueva praxis en la enseñanza de la matemática. Revista Andina de Educación, 12.

Palacios, D. E., Villa, H. E., \& Contreras, B. A. (2018). El álgebra geométrica como herramienta fundamental en el proceso de factorización polinómica. Medellín: Universidad Cooperativa de Colombia.

Pedraza, L., \& Valbuena, S. (2014). Plataforma móvil con realidad aumentada para enseñanza de los cálculos. Universidad de Manizales, 12.

Pulgarin, C., Sauceda, J., \& Matute, L. (2019). El álgebra geométrica en el aprendizaje de la factorización de trinomios cuadráticos. Universidad Cooperativa De Colombia, 25.

Rivera, D. (2020). Enseñanza de la factorización a partir de la relación entre álgebra y geometría. Medellín: Universidad Nacional de Colombia.

Sánchez, M. J. H., Villalba, C. I. C., Armijos, H. E. M., Requelme, S. E. R., \& Farías, J. J. C. (2021). El Crédito de Desarrollo Humano como estrategia de la Economía Popular y Solidaria para combatir la pobreza. Visionario Digital, 5(1), 52-69. 
Salinas, P., González Mendivil, E., Quintero, E., Ríos, H., Ramírez, H., \& Morales, S. (2013). La realidad aumentada y el aprendizaje del cálculo. Instituto Tecnológico y de Estudios Superiores de Monterrey, 7.

Zuluaga A, J. M., Pérez Q, F., \& Gómez T, J. (2015). Matemáticas y TIC. Ambientes virtuales de aprendizaje en clase de Matemáticas. Universidad Nacional Autónoma de México, 17.

Zurita, C., Zaldívar, A., Sifuentes, A., \& Valle, R. (2020). Análisis crítico de ambientes virtuales de aprendizaje. Utopía y Praxis Latinoamericana, 15.

\section{Liencia}


El artículo que se publica es de exclusiva responsabilidad de los autores y no necesariamente reflejan el pensamiento de la Revista Alfa Publicaciones.

\section{Ciencia}

El artículo queda en propiedad de la revista y, por tanto, su publicación parcial y/o total en otro medio tiene que ser autorizado por el director de la Revista Alfa Publicaciones.
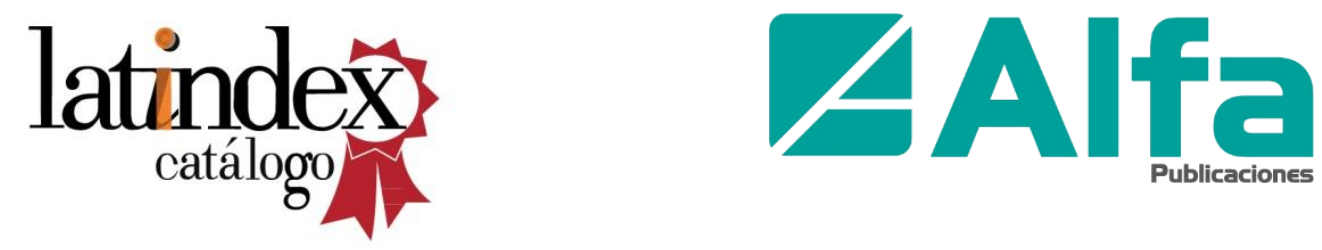

Indexaciones

d. DLatinREV 